\title{
Epidemiology and associated factors of polypharmacy in older patients in primary care: a northern Italian cross-sectional study
}

Giuliano Piccoliori ${ }^{1,2+}$, Angelika Mahlknecht ${ }^{1,3^{*}+}$ (D) Marco Sandri ${ }^{4}$, Martina Valentini ${ }^{2}$, Anna Vögele ${ }^{2}$, Sara Schmid ${ }^{2}$, Felix Deflorian², Adolf Engl ${ }^{1,2}$, Andreas Sönnichsen ${ }^{5}$ and Christian Wiedermann ${ }^{1,6}$

\begin{abstract}
Background: A precondition for developing strategies to reduce polypharmacy and its well-known harmful consequences is to study its epidemiology and associated factors. The objective of this study was to analyse the prevalence of polypharmacy (defined as $\geq 8$ prescribed drugs), of potentially inappropriate medications (PIMs) and major drug-drug interactions (DDIs) among community-dwelling general practice patients aged $\geq 75$ years and to identify characteristics being associated with polypharmacy.

Methods: This cross-sectional study is derived from baseline data (patients' demographic/biometric characteristics, diagnoses, medication-related data, cognitive/affective status, quality of life) of a northern-Italian cluster-RCT. PIMs and DDIs were assessed using the 2012 Beers criteria and the Lexi-Interact ${ }^{\oplus}$ database. Data were analysed using descriptive methods, Wilcoxon rank-sum tests, Fisher's exact tests and Spearman correlations.

Results: Of the eligible patients aged $75+, 13.4 \%$ were on therapy with $\geq 8$ drugs. Forty-three general practitioners and 579 patients participated in the study. Forty five point nine percent of patients were treated with $\geq 1$ Beers-listed drugs. The most frequent PIMs were benzodiazepines/hypnotics (19.7\% of patients) and NSAIDs (6.6\%). Sixty seven point five percent of patients were exposed to $\geq 1$ major DDI, 35.2\% to $\geq 2$ major DDls. Antithrombotic/anticoagulant medications (30.4\%) and antidepressants/antipsychotics (23.1\%) were the most frequently interacting drugs. Polypharmacy was significantly associated with a higher number of major DDIs (Spearman's rho 0.33, $p<0.001$ ) and chronic conditions (Spearman's rho 0.20, $p<0.001$ ), higher 5-GDS scores (thus, lower affective status) (Spearman's rho $0.12, p=0.003$ ) and lower EQ-5D-5L scores (thus, lower quality of life) (Spearman's rho $-0.14, p=0.001$ ). Patients' age/ sex, 6-CIT scores (cognitive status), BMI or PIM use were not correlated with the number of drugs.

* Correspondence: angelika.mahlknecht@am-mg.claudiana.bz.it

${ }^{\dagger}$ Giuliano Piccoliori and Angelika Mahlknecht contributed equally to this work.

${ }^{1}$ Institute of General Practice and Public Health, College of Health Care Professions, Lorenz Böhler- Straße 13, 39100 Bolzano, Italy

${ }^{3}$ Institute of General Practice, Family Medicine and Preventive Medicine, Paracelsus Medical University, Strubergasse 21, 5020 Salzburg, Austria

Full list of author information is available at the end of the article

(c) The Author(s). 2021 Open Access This article is licensed under a Creative Commons Attribution 4.0 International License, which permits use, sharing, adaptation, distribution and reproduction in any medium or format, as long as you give appropriate credit to the original author(s) and the source, provide a link to the Creative Commons licence, and indicate if changes were made. The images or other third party material in this article are included in the article's Creative Commons licence, unless indicated otherwise in a credit line to the material. If material is not included in the article's Creative Commons licence and your intended use is not permitted by statutory regulation or exceeds the permitted use, you will need to obtain permission directly from the copyright holder. To view a copy of this licence, visit http://creativecommons.org/licenses/by/4.0/ The Creative Commons Public Domain Dedication waiver (http://creativecommons.org/publicdomain/zero/1.0/) applies to the data made available in this article, unless otherwise stated in a credit line to the data. 


\begin{abstract}
(Continued from previous page)
Conclusions: The prevalence of polypharmacy, PIMs and major DDIs was considerable. Results indicate that physicians should particularly observe their patients with multiple conditions, reduced health and affective status, independently from other patients' characteristics. Careful attention about indication, benefit and potential risk should be paid especially to patients on therapy with specific drug classes identified as potentially inappropriate or prone to major DDIs in older persons (e.g., benzodiazepines, NSAIDs, protonic pump inhibitors, antithrombotics/anticoagulants, antidepressants/antipsychotics).
\end{abstract}

Trial registration: The cluster-RCT on which this cross-sectional analysis is based was registered with Current Controlled Trials Ltd. (ID ISRCTN: 38449870) on 2013-09-11.

Keywords: Polypharmacy, Inappropriate prescribing, Older adults, General practice, Drug interactions

\section{Background}

In Europe, chronic conditions are the leading cause of illness and disability and constitute large parts of healthcare costs [1]. Especially older-aged persons are not only likely to suffer from chronic conditions, but also to be affected from multiple diseases [2] with the consequence of reduced quality of life and impaired health outcomes. The phenomenon of multi-morbidity is highly prevalent in the older-aged population and raises complex needs of care as each condition can influence the clinical and therapeutic course of other concomitant pathologies [3]. This renders drug therapy challenging and entails the risk for polypharmacy.

A general consensus regarding the cut-off point defined as polypharmacy does not exist. In primary care, the most common cut-off is a use of $\geq 5$ drugs [4]. Depending on definition and setting, up to $54 \%$ of older persons have shown to be affected from polypharmacy [5]. This number rises up to $79 \%$ in residential care [6]. A cross-sectional analysis including 17 European countries and Israel found a polypharmacy rate of $26.3-39.9 \%$ among persons aged $65+$. The highest prevalence of polypharmacy was found in Portugal, Israel and the Czech Republic, whereas Switzerland, Croatia and Slovenia were the countries with the lowest polypharmacy rates [7].

In Germany, the age group 60+ was found to receive two thirds of all prescribed drugs [8]. In Italy, about two thirds of persons aged 65+ were prescribed four or more active agents per year and the older-aged population has shown to absorb nearly $2 / 3$ of the public pharmaceutical expenditure $[3,9]$.

Besides from a notable economic impact, polypharmacy has shown to entail several clinically harmful effects: increased risk for potentially inappropriate medications (PIMs) [10], under-use of appropriate medications, low patient compliance, drug-drug interactions (DDIs) [3], adverse drug events (ADEs) [11], functional decline [12], lower physical performance [13], hospitalisations due to ADEs (predictable from the known pharmacology of the prescribed drugs e.g. interactions, and therefore avoidable in 59-70\%) [14], short-time hospital readmissions [15], and even increased mortality [14].
Thus, polypharmacy has become a relevant public health issue and a major concern regarding patient safety in the field of medical treatment of older people.

The proportion of persons aged 65 or more years is increasing in the European population, especially the proportion of the oldest old ( 80 years or more) [16]. In the UK, one in 12 persons is estimated to be aged 80 or more years by 2039 [17]. In Italy, persons aged $\geq 65$ years are estimated to account for 33\% of the general population in 2051 [3]. At the same time, as drug use in older-aged patients is common, the consumption of drugs and the pharmaceutical expenditures are rising in Italy as well as in other countries [3].

Thus, polypharmacy will be of increasing clinical significance especially in general practice as the GPs are the major initiators and providers of drug prescriptions: although exact figures are not available, it may be assumed that $60-80 \%$ of prescriptions are initiated by GPs [18].

It is, therefore, crucial to study the epidemiology of polypharmacy and its associated factors in older-aged persons as a precondition for developing strategies to reduce polypharmacy and potentially harmful consequences in general practice. In the inpatient setting, efforts in this regard have been conducted since several years, e.g. the prospective REPOSI register which was started in 2008 with the aim to study the prevalence of polypharmacy and to improve medication appropriateness for older persons in Italy [9]; in European general practice, up to now, only few studies investigating the prevalence and predictors of polypharmacy have been conducted [19].

We therefore initiated the cluster-randomised controlled trial (RCT) 'PRIMA' (Polypharmacy in chronic diseases Reduction of Inappropriate Medication and Adverse drug events in older populations) (2014-2016) with the objective to investigate the impact of an intervention aiming at reducing polypharmacy on mortality/hospitalisation. ${ }^{1}$

\footnotetext{
${ }^{1}$ In contrast to the European multicenter trial PRIMA-eDS [19], the here presented PRIMA study was limited to a northern Italian region and applied medication reviews provided by three experts (a specialist in internal medicine, a clinical pharmacist and an EbM expert) as intervention. The data generated by the independent Italian PRIMA study were not part of the PRIMA-eDS trial.
} 
In this article, we present the cross-sectional analysis of the epidemiological baseline data of the RCT with the aim

- To analyse the prevalence of polypharmacy (defined as $\geq 8$ prescribed drugs), PIMs and DDIs among community-dwelling general practice patients aged $\geq 75$ years in a northern Italian region

- To detect associated factors with polypharmacy in general practice.

The cut-off of $\geq 8$ drugs was chosen for the underlying cluster-RCT as the sample-size calculation was based on a previous study [20] where the participating patients were treated on average with a corresponding number of medications.

\section{Methods}

\section{Study design, setting and population}

The cross-sectional study was conducted in the primary care setting in the province of Bolzano (Italy) and involved GPs and older-aged community-living patients.

\section{Recruitment}

All 270 active GPs listed in the Chamber of Physicians of Bolzano were informed and invited by email and phone to participate.

The participating GPs identified eligible patients meeting the following inclusion criteria:

- Age $\geq 75$ years

- On therapy with $\geq 8$ prescribed active agents (excluding PRN-medications and OTC-drugs)

- Absence of terminal illness/radiation/chemotherapy

- Sufficient cognitive function to be able to give informed consent.

The patients were consecutively invited to participate by the GPs during routine visits in the GP office. All participating GPs and patients gave written informed consent.

\section{Data collection}

Data were collected by means of structured case report forms (CRFs). For the $n=39$ GPs using the electronic health record (EHR) Millewin ${ }^{\circ}$, an add-on module was programmed which filled in automatically all required patient data which were available in the EHR. The GPs checked these electronically generated CRFs and completed all missing data manually (e.g., the results of the questionnaires, see below).

The following parameters were collected:

- Patient s demographic data: age, sex
- Patient s diagnoses (ICD-9-coded)

- Current medication (International Non-proprietary Names) and daily dosage in milligrams

- Biometric and laboratory parameters: height, weight, BMI, blood pressure, creatinine, potassium

- Health-related quality of life, cognitive function and affective status were measured by the EQ-5D-5L [21], the 6-Item Cognitive Impairment Test (6-CIT) [22], and the 5-Item Geriatric Depression Scale (5-GDS) [23]. The questionnaires were handed out to the patients by the GPs who recorded the results in the case report forms.

Data were pseudonymised by the GPs. Afterwards, the electronic CRFs were forwarded by email to the research team via the add-on module.

\section{Data analysis}

The drug regimens of all participating patients were assessed by two members of the project team (one GP, not included as study participant, and one student of pharmacology) regarding PIMs using the 2012 Beers criteria (Italian Version) [24, 25] and DDIs using the Lexi-Interact ${ }^{\oplus}$ database [26]. Only potentially severe DDIs were considered: categories $\mathrm{D}=$ consider drug modification, and $\mathrm{X}=$ avoid combination.

Values obtained from EQ-5D-5L were converted into the $E Q-5 D$ index (single value per patient; maximum = $1=$ full health) by using the German EQ-5D-5L Crosswalk Value Set [27] as no country-specific value set was available for Italy [28] and Germany most closely approximates to the investigated northern Italian region ${ }^{2}$ [29].

Statistical analysis was performed by an independent statistician via Stata 16.1 (StataCorp. 2019. Stata Statistical Software: College Station, TX). Categorical variables were summarised as absolute and relative frequencies, while numerical variables as median and interquartile range (IQR), as appropriate.

Wilcoxon rank-sum test and Fisher's exact test were used to compare the distribution of continuous and categorical variables between study groups, respectively. Spearman correlations were used to assess associations between variables (crude and adjusted for age, gender, number of chronic conditions, cognitive function) [30]. Moreover, logistic regression models were applied for the estimation of unadjusted and adjusted odds ratios (ORs) as a measure of the association between hyperpolypharmacy (defined as the use of $\geq 10$ drugs) and various study variables $[12,13]$. All tests were two-sided, the level of statistical significance was set at $p<0.05$.

\footnotetext{
${ }^{2}$ The EQ-5D user guide recommends using value sets of a country that most closely approximates to the country of interest in case of absence of a country-specific value set [29].
} 
Missing data: Demographical data of GPs/patients, diagnoses and medication-related data were complete. Laboratory values were not available for all patients; in case of missing values, a listwise deletion was applied (individuals with missing data were excluded from analysis of laboratory values).

\section{Results}

\section{Study participants, conditions and drug use}

Of 270 invited GPs, 43 (15.9\%) participated in the study.

The participating GPs treated 71,014 patients overall and 8015 patients aged $\geq 75$ years. Of these, 1075 patients (13.4\%) were on regular therapy with $\geq 8$ drugs and thus eligible. The percentage of patients on polypharmacy (on therapy with $\geq 8$ drugs) among those aged $75+$ varied between 5 and $37 \%$ per GP.

A total of 579 patients (53.9\% of the eligible patients) took part in the study. Characteristics of the study participants are summarised in Table 1.

The participating patients had a total number of 3143 diagnoses of chronic diseases and were treated overall with 5614 prescriptions. A total of 376 patients $(64.9 \%$ of the participating patients) had $\geq 5$ diagnoses of chronic conditions, 249 patients (43\% of the investigated patients) used $\geq 10$ drugs. The most frequent diagnoses and used drug classes are shown in Table 2.

\section{PIMs and DDIs}

PIMs: 341 drugs (6.1\% of all prescribed drugs) were classified as PIM according to the Beers criteria; 266 patients (45.9\% of all participating patients) had at least one PIM.

DDIs: 776 severe DDIs were retrieved with 182 different drugs being involved in at least one severe DDI. In total, 1276 active agents were involved in the 776 DDIs (22.7\% of all prescribed drugs); 391 patients $(67.5 \%$ of all participating patients) had at least one severe DDI (Tables 3 and 4).

\section{Polypharmacy, PIMs, DDIs and associated factors}

The strongest significant associations $(\mathrm{p}<0.001)$ were found between the number of drugs assumed and the number of severe DDIs (Spearman's rho: 0.33) respectively the number of chronic conditions (Spearman's rho: 0.20). Weaker significant associations were found between higher numbers of drugs and a lower EQ-5D-index/EQ$V A S$ score (inverse association; thus, lower health-related quality of life) and a higher 5-GDS score (thus, depressive status). Similar associations were shown for patients with a use of $\geq 10$ drugs (hyperpolypharmacy). The adjusted associations did not differ substantially from the unadjusted analyses (Table 5).

The number of drugs did not show a significant association with patient's age, patients' sex, BMI, cognitive
Table 1 Characteristics of the participating GPs $(n=43)$ and patients $(n=579)$

\begin{tabular}{|c|c|c|}
\hline \multicolumn{3}{|l|}{ Participating GPs } \\
\hline \multirow[t]{2}{*}{ Age } & & Median (IQR) \\
\hline & & $56(49-61)$ \\
\hline Sex & n GPs & $\%$ of participating GPs \\
\hline Male & 31 & $72.1 \%$ \\
\hline Female & 12 & $27.9 \%$ \\
\hline Location of GP office & n GPs & $\%$ of participating GPs \\
\hline Urban area & 21 & $48.8 \%$ \\
\hline Rural area & 22 & $51.2 \%$ \\
\hline \multicolumn{3}{|l|}{ Participating patients } \\
\hline \multirow[t]{2}{*}{ Age } & & Median (IQR) \\
\hline & & $81(78-85)$ \\
\hline Sex & $\mathrm{n}$ patients & $\%$ of participating patients \\
\hline Male & 230 & 39.7 \\
\hline Female & 349 & 60.3 \\
\hline \multicolumn{2}{|c|}{ Biometric and laboratory parameters } & Median (IQR) \\
\hline \multicolumn{2}{|l|}{ BMI $\left[\mathrm{kg} / \mathrm{m}^{2}\right]$} & $26.5(23.8-29.4)$ \\
\hline \multicolumn{2}{|c|}{ Systolic blood pressure [mmHg] } & $135(125-143)$ \\
\hline \multicolumn{2}{|c|}{ Diastolic blood pressure [mmHg] } & $77(70-80)$ \\
\hline \multicolumn{2}{|l|}{ Creatinine $[\mathrm{mg} / \mathrm{dll}]$} & $1.1(0.9-1.3)$ \\
\hline \multicolumn{2}{|l|}{ Potassium [mmol/l] } & $4.3(4.0-4.7)$ \\
\hline \multicolumn{2}{|c|}{ Health-related quality of life } & Median (IQR) \\
\hline \multicolumn{2}{|l|}{ EQ-5D-5L index } & $0.813(0.716-0.909)$ \\
\hline \multicolumn{2}{|l|}{ EQ-VAS score } & $60.0(50.0-80.0)$ \\
\hline Cognitive impairment & $\mathrm{n}$ patients & $\%$ of participating patients \\
\hline 6 -CIT score $\geq 8$ points & 158 & $27.3 \%$ \\
\hline Affective impairment & $\mathrm{n}$ patients & $\%$ of participating patients \\
\hline 5-GDS score $\geq 2$ points & 170 & $29.4 \%$ \\
\hline
\end{tabular}

GPs General practitioners, IQR Interquartile range, $B M I$ Body mass index, EQ-5D 5 -Item questionnaire measuring health-related quality of life, VAS Visual analogue scale, 6-CIT 6-Item Cognitive Impairment Test, 5-GDS 5-Item Geriatric Depression Scale

function, GP's sex and geographical location of the GP office (rural/urban area). Also, the number of drugs did not correlate with the number of PIMs.

In addition, no significant associations were found between the number of PIMs respectively DDIs and patients' age, patients' sex and number of chronic conditions (Table 5).

The following conditions were associated with higher numbers of drugs: arthrosis, diabetes mellitus, coronary heart disease, COPD (Table 6). Table 6 also shows the association between number of drugs and the most common drug classes.

\section{Discussion}

A summary of the main results of the study is provided in Table 7. 
Table 2 Frequencies of chronic conditions and drug use among the participating patients $(n=579)$

\section{Chronic conditions}

Number of diagnoses (total)

3143

\section{Most frequent diagnoses}

Hypertension

Arthrosis

Diabetes mellitus ||

Dyslipidaemia

Atrial fibrillation

Coronary heart disease

Osteoporosis

Depression

Benign prostatic hypertrophy

Gastro-oesophageal reflux disease

Chronic heart failure

Chronic obstructive pulmonary disease (COPD)

Chronic renal failure

Hyperuricaemia / gout

Hypothyroidism

Insomnia

Cerebrovascular disease / dementia

\section{Drug use}

\section{Number of drugs (total)}

5614

Number of drugs per patient

$8-9$ drugs

$\geq 10$ drugs

Most frequently used drug classes per patient ${ }^{a}$

ARBs + ACE-inhibitors

PPIS

Statins

Platelet-aggregation inhibitors

Beta-blockers

Minor diuretics (predominantly hydrochlorothiazide)

CCBs

Loop diuretics (predominantly Furosemide)

Vitamins (predominantly vit. D)

Antidepressants + antipsychotics

Oral anticoagulants

Anxiolytics/hypnotics (Benzodiazepines + Zolpidem)

Dietary supplements (predominantly Calcium)

Oral antidiabetic drugs

Antiasthmatic agents incl. Beta-adrenergics + anticholinergics

Opioids

Analgesics - Paracetamol

\begin{tabular}{|c|c|c|}
\hline Median (IQR) & Min & Max \\
\hline $5(4-6)$ & 1 & 14 \\
\hline n patients & $\%$ of participating patients & \\
\hline 490 & $84.6 \%$ & \\
\hline 266 & $45.9 \%$ & \\
\hline 220 & $38.0 \%$ & \\
\hline 202 & $34.9 \%$ & \\
\hline 179 & $30.9 \%$ & \\
\hline 175 & $30.2 \%$ & \\
\hline 157 & $27.1 \%$ & \\
\hline 124 & $21.4 \%$ & \\
\hline 110 & $19.0 \%$ & \\
\hline 98 & $16.9 \%$ & \\
\hline 93 & $16.1 \%$ & \\
\hline 81 & $14.0 \%$ & \\
\hline 73 & $12.6 \%$ & \\
\hline 65 & $11.2 \%$ & \\
\hline 64 & $11.1 \%$ & \\
\hline 60 & $10.4 \%$ & \\
\hline $58(54+4)$ & $10.0 \%(9.3 \%+0.7 \%)$ & \\
\hline Median (IQR) & Min & Max \\
\hline $9(8-11)$ & 8 & 20 \\
\hline n patients & $\%$ of participating patients & \\
\hline 330 & $57.0 \%$ & \\
\hline 249 & $43.0 \%$ & \\
\hline n patients & $\%$ of participating patients & \\
\hline $481(251+230)$ & $83.1 \%(43.4 \%+39.7 \%)$ & \\
\hline 320 & $55.3 \%$ & \\
\hline 319 & $55.1 \%$ & \\
\hline 311 & $53.7 \%$ & \\
\hline 306 & $52.8 \%$ & \\
\hline 273 & $47.2 \%$ & \\
\hline 248 & $42.8 \%$ & \\
\hline 244 & $42.1 \%$ & \\
\hline 227 & $39.2 \%$ & \\
\hline 215 & $37.1 \%$ & \\
\hline 190 & $32.8 \%$ & \\
\hline 173 & $29.9 \%$ & \\
\hline 171 & $29.5 \%$ & \\
\hline 156 & $26.9 \%$ & \\
\hline 134 & $23.1 \%$ & \\
\hline 127 & $21.9 \%$ & \\
\hline 126 & $21.8 \%$ & \\
\hline
\end{tabular}


Table 2 Frequencies of chronic conditions and drug use among the participating patients $(n=579)$ (Continued)

\begin{tabular}{lll}
\hline Thyroid hormones & 114 & $19.7 \%$ \\
Corticosteroids & 106 & $18.3 \%$ \\
NSAIDs + COX-2-inhibitors (Coxibe) & 88 & $15.2 \%$
\end{tabular}

IQR Interquartile range, Min Minimum, Max Maximum, COPD Chronic obstructive pulmonary disease, ARBs Angiotensin II receptor antagonists, $A C E$ Angiotensin converting enzyme, PPIs Proton pump inhibitors, CCBs Calcium channel blockers, NSAIDs Non-steroidal anti-inflammatory drugs, COX Cyclooxygenase

${ }^{a}$ If one patient was assuming two active agents from the same drug class, this was counted as one drug class

\section{Drug use, prevalence of polypharmacy and most common drug classes}

The number of drugs used within our study cohort was high: on average, patients took nine active agents per day. A similar degree of drug consumption was found also in other studies with a comparable setting [31]. Yet, also studies including not only patients on polypharmacy detected high average drug numbers among older adults (eight prescriptions) [32, 33].

Also the prevalence of polypharmacy in our study was considerable: $13.4 \%$ of general practice patients aged $75+$ were on therapy with $\geq 8$ drugs. This rate, although being not always fully comparable to previous studies due to different definitions of polypharmacy, can however be considered to lie within the range reported by others. A northern Italian study found that $48 \%$ of general practice patients aged 65+ were using 5-9 drugs (polypharmacy) and $10 \%$ used $\geq 10$ drugs (hyperpolypharmacy) [34]. Similar results were reported by a Swedish cohort study [35] and by an Italian population study [11]. The rate of patients using $\geq 10$ drugs in our sample (43\%, Table 2 ) seems much higher; however, as we included only patients taking $\geq 8$ drugs and were not able to stratify the use of drugs within the whole older-aged population, this number is not comparable to the hyperpolypharmacy rates detected by others.

A study that used the same cut-off as in our cohort ( $\geq 8$ drugs) detected a sharply higher prevalence of polypharmacy (50\%), however, the study had been conducted in the inpatient setting [36]. An Austrian study assessing hospital-admitted patients aged $75+$ and on therapy with $\geq 7$ drugs found an even higher prevalence of polypharmacy of 58\% [37].

In general, as studies often differ in terms of definition of polypharmacy, investigated age groups and settings, comparability between studies is limited. Moreover, results from studies with comparable cut-offs also differ largely. A northern Italian study using administrative databases of the local health authorities detected a rate of polypharmacy ( $\geq 5$ drugs) of $17 \%$ in patients aged $65+$ [38] while an Italian population study including all drugs reimbursed by the National Healthcare System (NHS) showed $49 \%$ of people aged $65+$ to be on therapy with 5-9 drugs and $11 \%$ with $\geq 10$ drugs [11].

Table 3 Frequency of potentially inappropriate medications and drug-drug interactions

\begin{tabular}{|c|c|c|c|c|}
\hline \multicolumn{5}{|c|}{ Potentially inappropriate drugs according to the Beers-list [24] } \\
\hline Number of PIMs (total) & Median (IQR) & Mean $\pm S D^{a}$ & Min & Max \\
\hline 341 (6.1\% of all prescribed drugs) & $0(0-1)$ & $0.6 \pm 0.8$ & 0 & 4 \\
\hline n PIMs per patient & n patients & \multicolumn{3}{|c|}{$\%$ of participating patients } \\
\hline 0 & 313 & \multicolumn{3}{|l|}{$54.1 \%$} \\
\hline 1 & 207 & \multicolumn{3}{|l|}{$35.7 \%$} \\
\hline$\geq 2$ & 59 & \multicolumn{3}{|l|}{$10.2 \%$} \\
\hline TOTAL patients with $\geq 1$ Beers-listed drug & 266 & \multicolumn{3}{|l|}{$45.9 \%$} \\
\hline \multicolumn{5}{|l|}{ D or $\mathbf{X}^{\mathrm{b}}$ drug-drug interactions [26] } \\
\hline Number of DDls (total) & Median per patient (IQR) & \multicolumn{2}{|l|}{ Min } & Max \\
\hline 776 & $1(0-2)$ & \multicolumn{2}{|l|}{0} & 8 \\
\hline $\mathrm{n} \mathrm{D}$ or $\mathrm{X}^{\mathrm{b}}$ interactions per patient & n patients & \multicolumn{3}{|c|}{$\%$ of participating patients } \\
\hline 0 & 188 & \multicolumn{3}{|l|}{$32.5 \%$} \\
\hline 1 & 187 & \multicolumn{3}{|l|}{$32.3 \%$} \\
\hline 2 & 103 & \multicolumn{3}{|l|}{$17.8 \%$} \\
\hline$\geq 3$ & 101 & \multicolumn{3}{|l|}{$17.4 \%$} \\
\hline TOTAL patients with $\geq 1$ D or $X$ interaction & 391 & \multicolumn{3}{|l|}{$67.5 \%$} \\
\hline
\end{tabular}

PIMs Potentially inappropriate drugs, IQR Interquartile range, SD Standard deviation, Min Minimum, Max Maximum, DDIs Drug-drug interactions

${ }^{\text {a }}$ As the median was 0 , in this case also the mean per patient is reported to provide better comprehensibility

${ }^{b}$ Drug-drug interactions: category $\mathrm{D}=$ consider drug modification, category $\mathrm{X}=$ avoid combination [26] 
Table 4 Most common Beers-listed drug classes, DDls, and drug classes involved in DDIs

\begin{tabular}{|c|c|c|c|}
\hline Beers-listed drug classes [24] & n drugs & $\%$ of $n=579$ patients & Possible clinical consequences \\
\hline Benzodiazepines + Zolpidem & 114 & $19.7 \%$ & cognitive impairment, delirium, falls/fractures \\
\hline NSAIDs + COX-2-inhibitors (Coxibe) & 38 & $6.6 \%$ & bleeding, nephrotoxicity \\
\hline Antiarrhythmics & 37 & $6.4 \%$ & toxicity, QT alteration \\
\hline Alpha-blockers & 30 & $5.2 \%$ & hypotension \\
\hline Diuretics + Spironolacton & 29 & $5.0 \%$ & $\uparrow$ potassium \\
\hline CCBs & 26 & $4.5 \%$ & myocardial ischemia, hypotension \\
\hline Antithrombotic drugs, mainly Ticlopidine & 22 & $3.8 \%$ & toxicity, safer alternatives available \\
\hline Antidepressants and antipsychotics & 20 & $3.5 \%$ & $\begin{array}{l}\text { anticholinergic effects, cardio-vascular events, } \\
\text { hyponatriaemia }\end{array}$ \\
\hline Cardiac glycosides & 13 & $2.2 \%$ & toxicity, safer alternatives available \\
\hline $\mathrm{D}^{\mathrm{a}}$ or $\mathrm{X}^{\mathrm{a}}$ drug-drug interactions [26] & n DDls & $\%$ of $n=579$ patients & Possible clinical consequences \\
\hline Acenocoumarol/Warfarin - Allopurinol & 32 & $5.5 \%$ & $\uparrow$ risk of bleeding \\
\hline Alendronate - Calcium carbonate & 29 & $5.0 \%$ & $\downarrow$ absorption of alendronate \\
\hline Amlodipine/Lercanidipine - Simvastatin & 27 & $4.7 \%$ & myopathy/rhabdomyolysis \\
\hline Levothyroxine - Calcium carbonate & 26 & $4.5 \%$ & $\downarrow$ absorption of levothyroxine \\
\hline Allopurinol - Ramipril/Lisinopril/Enalapril & 21 & $3.6 \%$ & allergic reactions to allopurinol \\
\hline Bisoprolol - Tamsulosin & 18 & $3.1 \%$ & hypotension \\
\hline Acenocoumarol/Warfarin - Acetylsalicylic acid & 16 & $2.8 \%$ & $\uparrow$ risk of bleeding \\
\hline Ibuprofen/Diclofenac - Acetylsalicylic acid & 15 & $2.6 \%$ & $\uparrow$ risk of bleeding \\
\hline Acetylsalicylic acid - Etoricoxib & 12 & $2.1 \%$ & $\uparrow$ toxicity of etoricoxib, bleeding \\
\hline Clopidogrel - Pantoprazole & 11 & $1.9 \%$ & $\downarrow$ effectiveness of clopidogrel \\
\hline Bisoprolol - Doxazosin & 9 & $1.6 \%$ & hypotension \\
\hline Ibuprofen - Warfarin & 9 & $1.6 \%$ & $\uparrow$ risk of bleeding \\
\hline Ibuprofen - Furosemide & 8 & $1.4 \%$ & nephrotoxicity \\
\hline Prednison - Calcium carbonate & 7 & $1.2 \%$ & $\downarrow$ absorption of prednison \\
\hline Drug classes involved in $\mathrm{D}^{\mathrm{a}}$ or $\mathrm{X}^{\mathrm{a}} \mathrm{DDls}$ & n DDls & $\%$ of $n=579$ patients & \\
\hline Antithrombotic / anticoagulant drugs & 176 & $30.4 \%$ & \\
\hline Antidepressants / antipsychotics & 134 & $23.1 \%$ & \\
\hline Calcium carbonate & 77 & $13.3 \%$ & \\
\hline Beta-blockers & 74 & $12.8 \%$ & \\
\hline Alpha-blockers & 72 & $12.4 \%$ & \\
\hline Statins & 64 & $11.1 \%$ & \\
\hline NSAIDs + COX-2-inhibitors (Coxibe) & 62 & $10.7 \%$ & \\
\hline ARBs/ACE-inhibitors & 61 & $10.5 \%$ & \\
\hline CCBs & 54 & $9.3 \%$ & \\
\hline Drugs for gout treatment-Allopurinol & 48 & $8.3 \%$ & \\
\hline
\end{tabular}

NSAIDs Non-steroidal anti-inflammatory drugs, COX Cyclooxygenase, CCBs Calcium channel blockers, DDIs Drug-drug interactions, ARBs Angiotensin II receptor antagonists, ACE Angiotensin converting enzyme

a Drug-drug interactions: category $\mathrm{D}=$ consider drug modification, category $\mathrm{X}=$ avoid combination [26]

These notable differences within the same country suggest that polypharmacy rates depend not only on setting but may also vary according to local-regional differences and the used data sources. Moreover, differences between polypharmacy rates can even be considerable between single physicians: In our study, the prevalence of polypharmacy ranged from 5 to $37 \%$ among the participating GPs. A similar phenomenon was described in other studies [39]. Factors that determine variations in prescribing rates are not well understood; demographic characteristics of patients and GPs themselves may play a role as well as the GP-patient relationship and/or GP-related factors (different prescribing patterns based on training, practice organisation etc.) [39]. 
Table 5 Polypharmacy, PIMs and DDIs and associated factors (bold numbers = significant results)

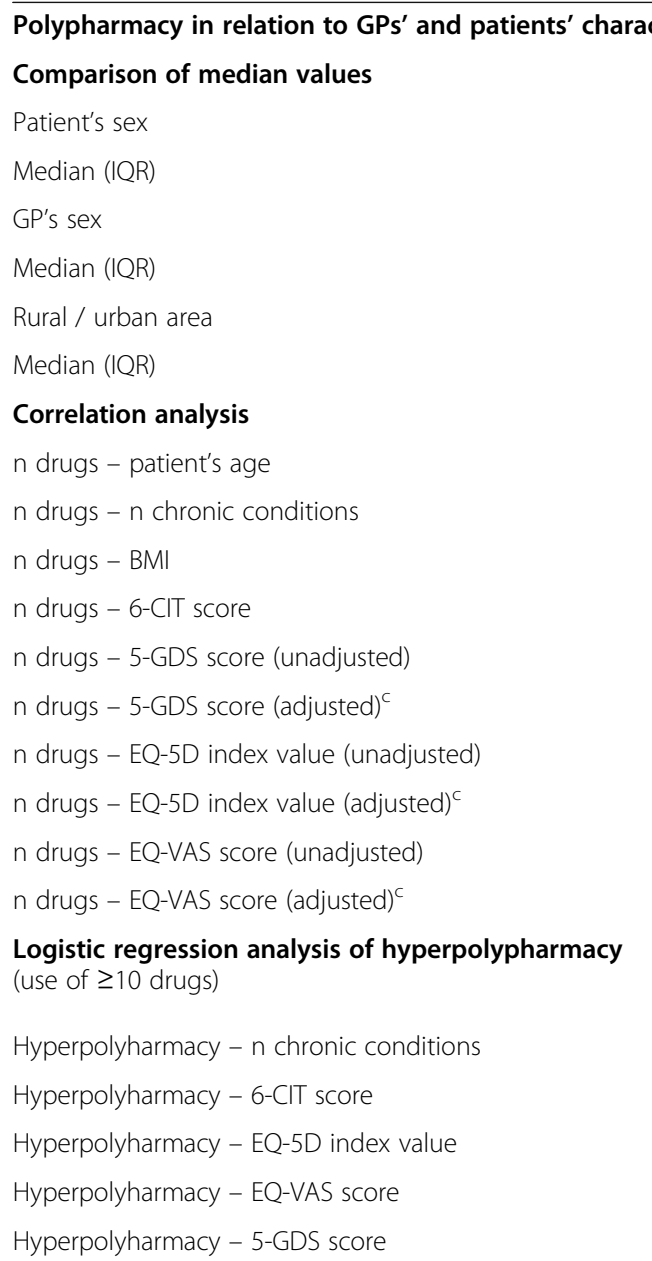

PIM use (Beers-listed drugs) in relation to patient's characteristics
Number of drugs

Male Female

$9(8-10) \quad 9(8-11)$

Male Female

$9(8-10) \quad 9(8-11)$

Rural Urban

$9(8-10) \quad 9(8-10)$

Correlation coefficient

0.06

0.20

0.01

0.03

0.12

0.10

$-0.14$

$-0.09$

$-0.14$

$-0.11$

\section{Crude analysis}

OR

1.26

1.01

0.85

0.86

1.19

p-value

$<0.001$

0.692

$<0.001$

0.007

0.021 p-value

$0.319^{a}$

$0.818^{a}$

$0.655^{a}$

p-value

$0.146^{b}$

$<0.001^{b}$

$0.817^{b}$

$0.524^{b}$

$0.003^{b}$

$0.017^{c}$

$0.001^{b}$

$0.034^{c}$

$0.001^{b}$

$0.006^{c}$

Adjusted analysis (by age, sex, n conditions, 6-CIT)

$\mathrm{OR}$

$p$-value

1.23

$<0.001$

0.583

0.008

0.035

0.065

p-value

Male

Female

$51.7 \%$

$55.6 \%$

$0.385^{d}$

$36.1 \%$

$35.5 \%$

$12.2 \%$

$8.9 \%$

Correlation coefficient

$p$-value

$0.358^{b}$

$0.252^{b}$

$0.089^{b}$

$0.156^{c}$

p-value

Male

Female

$30.4 \%$

$33.8 \%$

$0.374^{d}$

$\%$ of patients with $0 \mathrm{D} / \mathrm{X}$ drug-drug interaction 
Table 5 Polypharmacy, PIMs and DDIs and associated factors (bold numbers = significant results) (Continued)

\begin{tabular}{lll}
\hline Correlation analysis & Correlation coefficient & $p$-value \\
$\mathrm{n}$ D/X-DDls - patient's age & 0.04 & $0.348^{\mathrm{b}}$ \\
$\mathrm{n}$ D/X-DDls - $\mathrm{n}$ chronic conditions & 0.05 & $0.220^{\mathrm{b}}$ \\
$\mathrm{n}$ D/X-DDls $-\mathrm{n}$ drugs (unadjusted) & 0.33 & $<\mathbf{0 . 0 0 1 ^ { \mathrm { b } }}$ \\
$\mathrm{n}$ D/X-DDls $-\mathrm{n}$ drugs (adjusted) ${ }^{c}$ & 0.33 & $<\mathbf{0 . 0 0 1 ^ { c }}$ \\
\hline
\end{tabular}

PIM Potentially inappropriate medication, DDIs Drug-drug interactions, GP General practitioner, IQR Interquartile range, BMI Body mass index, 6-CIT 6-Item Cognitive Impairment Test, 5-GDS 5-Item Geriatric Depression Scale, EQ-5D 5-Item questionnaire measuring health-related quality of life, VAS Visual analogue scale, OR Odds Ratio

${ }^{a}$ Wilcoxon rank-sum test

${ }^{\mathrm{b}}$ Spearman correlation unadjusted (crude)

c Spearman correlation adjusted by age, sex, number of chronic conditions, cognitive function (6-CIT)

${ }^{\mathrm{d}}$ Fisher's exact test

The most often used drug classes in our study were in accordance with previous investigations [33, 40]. Concordantly to the most prevalent chronic condition hypertension and in consistence with the respective recommended first-line treatment [41] the most frequently prescribed drug classes were ARBs/ACE-inhibitors $(83 \%)$ with ARBs being slightly more frequently used than ACE-inhibitors (Table 2). ACE-inhibitors are recommended to be used prior to $\mathrm{ARBs}$ due to their lower costs and similar benefit [41] except in case of contraindications.

About half of the investigated patients received PPIs, statins, antithrombotic agents, beta-blockers and/or minor diuretics. 40-30\% were treated with CCBs, loop diuretics, vitamin $\mathrm{D}$, antidepressants, oral anticoagulants, benzodiazepines and/or calcium.

The large European multicentre trial PRIMA-eDS which included primary care patients from UK, Austria, Italy and Germany and used similar inclusion criteria (patients aged $75+$ and taking $\geq 8$ drugs) reported the same top five drug classes. As in our cohort, ACEinhibitors/ARBs were the most frequent therapeutic subgroup (80\%), followed by statins which were prescribed more often than in our sample (64\% vs. $55 \%$ ); the prescription rates of PPIs, beta-blockers and antithrombotic agents were approximately similar to our study [19].

Other studies reported statins to be the most used drug class in older patients [42, 43] respectively diuretics [37] respectively beta-blockers [31]. In the latter study, NSAIDs (28\%) were prescribed considerably more often than in our cohort (15\%), however, as we did not include OTC-drugs and some (however only low-dose) NSAIDs are available without prescription in Italy, the use of NSAIDs in our study could slightly have been underestimated.

The use of dietary supplements within our sample was high: $39 \%$ of patients were treated with vitamin D and $30 \%$ with calcium. A US population study found even $64 \%$ of older persons to regularly use dietary supplements [43]. In general, evidence regarding dietary supplements is not convincing; however, some research suggested possible beneficial effects of vitamin D on falls and cognitive function in older adults which may have contributed to its frequent and increasing use [43].

\section{Chronic conditions}

The included patients were multimorbid with on average five chronic conditions per patient. Nearly two thirds $(65 \%)$ of the included patients were diagnosed with $\geq 5$ chronic conditions.

Arterial hypertension was by far the most frequent diagnosis (85\% of patients), followed by arthrosis, diabetes mellitus type 2, dyslipidaemia, atrial fibrillation and coronary heart disease. About one fifth of the investigated patients suffered from depression. The ranking of the most prevalent conditions was comparable to other polypharmacy studies $[19,34]$.

Population studies of older patients with similar mean age [2] found a considerably higher prevalence of dementia (21\%) compared to our sample. This may be related to (a) a true lower rate of polypharmacy among dementia patients which was confirmed also by other research [44] due to lower life expectancy and less benefit of multiple pharmacological treatment, or to (b) a possible under-representation of dementia in our study cohort, possibly also due to the fact that severe cognitive dysfunction was an exclusion criterium (only four of 579 patients were explicitly diagnosed with dementia, Table 2 ).

\section{Potentially inappropriate medications}

Nearly half of patients $(46 \%)$ in our cohort were treated with at least one PIM, 10\% received $\geq 2$ Beers-listed drugs. On average, one out of two patients received PIMs. The most frequent PIM classes were benzodiazepines/hypnotics (20\% of patients) and NSAIDs (7\%), followed by antiarrhythmics and alpha-blockers. Similar distributions of PIM classes were detected by other studies [34, 37, 45] while PIM prevalences varied notably in previous studies on community-dwelling older persons (12- 69\%) [32, 45]. A study using the 2015 Beers criteria reported $46 \%$ of older-aged primary care patients to be treated with $\geq 2$ Beers-listed drugs [45]; this is more than 
Table 6 Polypharmacy and its association with conditions and drug classes (bold numbers = significant results)

\begin{tabular}{|c|c|c|c|}
\hline Polypharmacy in relation to the most frequent conditions & Median (IQR) & & $p$-value ${ }^{a}$ \\
\hline Chronic conditions & $\begin{array}{l}\mathrm{n} \text { drugs in patients } \\
\text { with the condition }\end{array}$ & $\begin{array}{l}\mathrm{n} \text { drugs in patients without } \\
\text { the condition }\end{array}$ & \\
\hline Hypertension & $9(8-11)$ & $9(8-10)$ & 0.081 \\
\hline Arthrosis & $10(8-11)$ & $9(8-10)$ & $<0.001$ \\
\hline Diabetes mellitus II & $9(8-11)$ & $9(8-10)$ & 0.004 \\
\hline Dyslipidaemia & $9(8-11)$ & $9(8-11)$ & 0.901 \\
\hline Atrial fibrillation & $9(8-11)$ & $9(8-11)$ & 0.721 \\
\hline Coronary heart disease & $10(8-11)$ & $9(8-10)$ & 0.006 \\
\hline Osteoporosis & $9(8-11)$ & $9(8-11)$ & 0.292 \\
\hline Depression & $9(8-11)$ & $9(8-11)$ & 0.051 \\
\hline Benign prostatic hypertrophy & $9(8-11)$ & $9(8-11)$ & 0.255 \\
\hline Gastro-oesophageal reflux disease & $9(8-10)$ & $9(8-11)$ & 0.885 \\
\hline Chronic heart failure & $9(8-11)$ & $9(8-11)$ & 0.443 \\
\hline Chronic obstructive pulmonary disease (COPD) & $10(8-12)$ & $9(8-10)$ & 0.016 \\
\hline Chronic renal failure & $9(8-11)$ & $9(8-11)$ & 0.158 \\
\hline Hyperuricaemia and/or gout & $9.5(9-11)$ & $9(8-11)$ & 0.148 \\
\hline Hypothyroidism & $9(8-11)$ & $9(8-11)$ & 0.472 \\
\hline Insomnia & $9(8-11)$ & $9(8-11)$ & 0.923 \\
\hline Cerebrovascular disease & $9(8-11)$ & $9(8-11)$ & 0.986 \\
\hline Polypharmacy in relation to the most frequent drug classes & Median (IQR) & & $p$-value ${ }^{a}$ \\
\hline Drug classes & $\begin{array}{l}\mathrm{n} \text { drugs in patients } \\
\text { with the drug class }\end{array}$ & $\begin{array}{l}\mathrm{n} \text { drugs in patients without } \\
\text { the drug class }\end{array}$ & \\
\hline ARBs/ACE-inhibitors & $9(8-11)$ & $9(8-10)$ & 0.137 \\
\hline PPIs & $9(8-11)$ & $9(8-10)$ & $<0.001$ \\
\hline Statins & $9(8-11)$ & $9(8-10)$ & 0.257 \\
\hline Platelet-aggregation inhibitors & $9(8-11)$ & $9(8-10)$ & 0.056 \\
\hline Beta-blockers & $9(8-11)$ & $9(8-10)$ & 0.461 \\
\hline Minor diuretics (predominantly hydrochlorothiazide) & $9(8-11)$ & $9(8-11)$ & 0.642 \\
\hline CCBs & $10(8-11)$ & $9(8-10)$ & 0.004 \\
\hline Loop diuretics (predominantly Furosemide) & $10(9-11)$ & $9(8-10)$ & $<0.001$ \\
\hline Vitamins (predominantly vit. D) & $10(8-11)$ & $9(8-10)$ & $<0.001$ \\
\hline Antidepressants & $10(9-12)$ & $9(8-10)$ & $<0.001$ \\
\hline Oral anticoagulants & $9(8-11)$ & $9(8-11)$ & 0.620 \\
\hline Anxiolytics/hypnotics (Benzodiazepines + Zolpidem) & $10(8-11)$ & $9(8-10)$ & $<0.001$ \\
\hline Dietary supplements (predominantly Calcium) & $10(9-11)$ & $9(8-10)$ & $<0.001$ \\
\hline Oral antidiabetic drugs & $9.5(8-11)$ & $9(8-10)$ & 0.018 \\
\hline Antiasthmatic agents, beta-adrenergics, anticholinergics & $10(9-12)$ & $9(8-10)$ & $<0.001$ \\
\hline Opioids & $10(9-12)$ & $9(8-10)$ & $<0.001$ \\
\hline Analgesics - Paracetamol & $9(8-11)$ & $9(8-11)$ & 0.385 \\
\hline Thyroid hormones & $10(8-11)$ & $9(8-10)$ & 0.009 \\
\hline Corticosteroids & $10(9-12)$ & $9(8-10)$ & $<0.001$ \\
\hline NSAIDs + COX-2-inhibitors (Coxibe) & $9(8-10)$ & $9(8-11)$ & 0.716 \\
\hline
\end{tabular}

IQR Interquartile range, COPD Chronic obstructive pulmonary disease, ARBs Angiotensin II receptor antagonists, ACE Angiotensin converting enzyme, PPIs Proton pump inhibitors, CCBs Calcium channel blockers, NSAIDs Non-steroidal anti-inflammatory drugs, COX Cyclooxygenase

${ }^{\text {a }}$ Wilcoxon rank-sum test 
Table 7 Summary of the main study findings

\begin{tabular}{|c|c|}
\hline \multirow[t]{3}{*}{ Drug use } & $13.4 \%$ of patients aged $\geq 75$ years were prescribed $\geq 8$ drugs \\
\hline & Median drug use among the included patients: 9 drugs \\
\hline & $\begin{array}{l}\text { Most commonly used drug classes: Angiotensin II receptor antagonists / ACE-inhibitors, protonic pump } \\
\text { inhibitors, statins, platelet-aggregation inhibitors, beta-blockers }\end{array}$ \\
\hline \multirow[t]{2}{*}{ Chronic conditions } & $64.9 \%$ of the participating patients had $\geq 5$ diagnoses of chronic conditions \\
\hline & Most frequent diagnoses: Arterial hypertension, arthrosis, diabetes mellitus II, dyslipidaemia, atrial fibrillation \\
\hline \multirow[t]{2}{*}{ PIMs } & 45.9\% of patients were treated with at least one PIM according to the 2012 Beers criteria \\
\hline & Most common PIMs: Benzodiazepines and Zolpidem, NSAIDs / COX-2-inhibitors (Coxibe), antiarrhythmics \\
\hline \multirow[t]{2}{*}{ DDIs } & $67.5 \%$ of patients were exposed to at least one major DDI \\
\hline & $\begin{array}{l}\text { Drug classes most frequently involved in major DDls: antithrombotic / anticoagulant drugs, antidepressants } \\
\text { / antipsychotics, calcium carbonate }\end{array}$ \\
\hline \multirow[t]{2}{*}{$\begin{array}{l}\text { Polypharmacy and } \\
\text { associated factors }\end{array}$} & $\begin{array}{l}\text { Significant correlations }(p<0.05) \text { : } \\
\text { - number of drugs - number of major DDIs (Spearman's rho: 0.33) } \\
\text { - number of drugs - number of chronic conditions (Spearman's rho: 0.20) } \\
\text { - number of drugs - reduced affective status (Spearman's rho: 0.12) } \\
\text { - number of drugs - reduced QoL / health status (Spearman's rho: -0.14) }\end{array}$ \\
\hline & $\begin{array}{l}\text { Conditions associated with higher numbers of drugs: } \\
\text { Arthrosis, diabetes mellitus II, coronary heart disease, COPD }\end{array}$ \\
\hline
\end{tabular}

ACE Angiotensin converting enzyme, PIMs Potentially inappropriate medications, NSAIDs Non-steroidal anti-inflammatory drugs, COX Cyclooxygenase, DDIs Drugdrug interactions, QoL Quality of life, COPD Chronic obstructive pulmonary disease

fourfold higher than in our cohort. The most frequently used Beers-listed drug classes were PPIs (46\% of patients), and, as in our sample, NSAIDs and benzodiazepines. PPIs were not detected as PIMs in our study since we used the 2012 Beers criteria (in the 2015 and later versions of the Beers criteria, PPIs were added as potentially inappropriate medications when used beyond eight weeks without clear indication, as evidence supported an association between long-term use of PPIs and Clostridium difficile infection and bone loss) [46]; however, it is noteworthy that only one fifth of patients in our cohort were diagnosed with gastro-oesophageal pathologies while 55\% received PPIs. This leads to the assumption that PPIs were probably inappropriately used also in a notable percentage of patients within our cohort. The fact that the Italian Medicines Agency (AIFA, Agenzia Italiana del Farmaco) stipulates a prescription of PPIs at the charge of the National Health Service in case of antiaggregant therapy with acetylsalicylic acid independently from gastrointestinal risk [47] had probably a relevant impact on the PPI prescription rates in our sample. The Italian multicenter prospective CRIME study found PPIs to be inappropriately prescribed in $30 \%$ of hospital-discharged older patients, however, also under-prescription of PPIs was found in $11 \%$ of patients in this cohort, particularly in older patients with higher numbers of comorbidities and drugs [48].

Benzodiazepines are frequently used in primary care although their risk of falls/fractures and cognitive impairment is well-known [49]. They were among the most common drug classes also in our cohort (30\%). Although we did not assess the specific indication for each administered drug, according to the most prevalent diagnoses it may be assumed that benzodiazepines were prescribed mostly for insomnia (10\% of patients) and/or depression (21\%). According to a German qualitative study [49], benzodiazepines are frequently prescribed also in case of insistent patient's demands in absence of serious or clear indications. Thus, GPs are faced with the challenge to address both medical recommendations and patient's expectations and to do so within a reasonable timeframe. On the other hand, a UK study showed that patients are often not adequately informed about the risks of benzodiazepines and that patients' willingness to attempt therapy withdrawal is given [50]. Therefore, increased patient information is required which can support the GPs' efforts regarding avoidance or withdrawal of inappropriate benzodiazepine prescriptions; this remains, however, difficult in case of drug dependency after long-term benzodiazepine use.

NSAIDs were prescribed to $15 \%$ of patients in our sample; in half of these cases, they were rated as inappropriate. This seems to be lower when compared to other studies [45]; however, NSAIDs have a well-known, high potential for DDIs and ADEs in older persons especially in case of comorbidities and concomitant corticosteroid or antiplatelet/anticoagulant medication and have also shown to be associated with major cardiovascular events independently from baseline cardiovascular risk [42]. For daily practice, it is recommended that NSAIDs should be possibly avoided in older patients [42]; however, complete avoidance is difficult, not least because of the frequent arthrosis-related disorders in this age group. Thus, thorough careful risk-benefit consideration is required. 
Overall, studies confirm the harmful impact of PIM use. A recent systematic review showed PIM use to be associated with increased mortality risk in older adults [51]. Therefore, and as PIMs were prescribed to nearly half of patients in our cohort, physicians' awareness towards more restrictive PIM use should be increased. Yet, in some individual cases, a valid indication for the use of a Beers-listed drug might be given, e.g. amiodarone in therapy-resistant arrhythmias [37].

\section{Drug-drug interactions}

About two thirds (68\%) of patients in our sample were exposed to at least one major interaction and more than one third had $\geq 2$ major interactions. $17 \%$ were exposed to $\geq 3$ major interactions. On average, every patient had one major DDI. These numbers show a high prevalence of DDIs and were largely confirmed also by other research [31, 37].

Other studies found higher average frequencies of DDIs in older-aged primary care patients on polypharmacy (1.5 DDIs per patient) respectively in a hospital setting (2.6 DDIs per patient) [2, 38]. In our sample, the most common EHR used by the participating GPs integrated a DDI-checking programme which alerted the physicians in case of clinically relevant DDIs at any drug prescription; this could have led a priori to a reduction of DDIs in our cohort.

In general, comparability of DDIs between studies is limited due to incongruent grading tools, different ways of reporting (all retrieved DDIs vs. 'clinically relevant'/ 'severe'/ 'high-risk' DDIs) and different drug availabilities and prescribing patterns across countries and settings [38]. However, the prevalence of DDIs in our cohort is still alarming despite EHR software use: a multivariate analysis found a significant association between exposition to $\geq 2$ severe DDIs and an increased risk of 3-monthsmortality [52].

In consistence with other studies [2], antithrombotic and anticoagulant medications were the most frequently interacting drugs (30\% of patients) followed by antidepressant and antipsychotic medications (23\%), calcium carbonate and beta-blockers (13\%). Regarding antithrombotic and anticoagulant drugs, which are characterised by a narrow therapeutic window and a high potential for DDIs [40], the most frequent interactions were with allopurinol, acetylsalicylic acid and NSAIDs. All these DDIs carry an increased risk of haemorrhagic complications [26]. Also calcium carbonate was commonly involved in major DDIs. Calcium interferes with the absorption of other active agents thus lowering their plasma level; this can, however, be avoided by separating the administration and/or dose adjustment [26].

The most common DDIs in our sample implicated commonly used drugs. These findings are hardly surprising; however, they suggest that GPs in daily practice should give more attention to combinations of frequently used drugs which may lead to avoidable ADEs. Thus, careful therapy monitoring in older patients with multiple conditions should be conducted regularly. On the other hand, also potentially severe DDIs can sometimes be accepted if the expected benefit of a combination therapy (e.g. double antiplatelet/anticoagulant therapy) outweighs the risk; this requires an appropriate and continuous patient monitoring and counseling [38].

\section{Polypharmacy and associated factors}

As in other studies $[19,34,35,40]$, multimorbidity was an important factor associated with polypharmacy (independently from patients' age and gender). Especially diabetes, coronary heart disease, COPD and arthrosis were associated with high numbers of drugs in our sample. Other authors reported cardiometabolic diseases and COPD $[9,44]$ respectively hypertension $[37,53]$, depression and pain $[37,53,54]$ as predictors of polypharmacy.

Most of these conditions are highly prevalent in olderaged persons and their evidence-based treatment often requires the administration of several drugs. Thus, multiple drug use may sometimes be unavoidable and polymedication is not always synonymous with an inappropriate overuse of drugs. E.g., a study in German nursing homes showed a postinterventional increase of medication appropriateness despite of unchanged numbers of drugs [6].

As in other research [37], we detected no association between polypharmacy and patients' age. Study findings in this regard are contradicting. Some research detected almost linear associations with the number of drugs remaining high among the oldest old [35, 40, 54, 55]. This was confirmed also by the Italian EPIFARM study [56]. In contrast, the Italian CRIME study found no variation of drug numbers across age groups [57]; this study included, however, inpatients of geriatric and internal medicine acute care wards. Other European studies found the age of $85+$ to be a protective factor against excessive polypharmacy $[19,58]$ and claimed as possible explanations (1) a real lower drug use in this age group because of reduced life expectancy, or (2) the possibility that patients exposed to excessive polypharmacy died already before reaching the very old age [19]. Also, reduced patients' compliance could have played a role.

Patients' sex was not linked to polypharmacy in our sample. Some studies found male sex [40, 58], other studies reported female sex $[37,54,55]$ to be associated with polypharmacy. Our results as well as previous findings [19] suggest that polypharmacy is an equivalent phenomenon in both male and female patients and should therefore be observed independently from patients' sex. 
No associations between cognitive status and polypharmacy were noted in our sample as well as in previous research [37], however, polypharmacy showed weak but significant associations with an impaired affective status and lower health-related quality of life and self-reported health status. The latter findings were confirmed by other studies $[19,54]$. Yet, our analyses do not allow an affirmation regarding causality: polypharmacy itself may impair quality of life and affective status (e.g. due to ADEs), or an impaired functional status (e.g. due to multimorbidity) may entail the risk for polypharmacy. We assume that both explanatory models may play a role. Consequently, when prescribing drug therapies, the GP has to pay particular attention to patients with impaired health and affective status.

In contrast to other studies [37], PIM use in our sample was not related to the number of drugs. Evidence regarding the association between PIM use and patients' age, gender and multimorbidity is contradicting [32, 45, 59]. E.g., a higher PIM use was found in patients with higher number of chronic conditions [32]; another study confirmed this only for males [59].

Polypharmacy in our cohort was significantly associated with the number of major DDIs. This was the strongest association (although it has still to be considered as weak) and has been confirmed by previous studies $[37,55,60]$. Evidence regarding associations between DDIs and other patient-related factors (which were missing in our cohort) is inconsistent; some research detected higher DDI prevalences in patients with older age [55], male sex [43, 55] respectively multimorbidity [60].

Most of the commonly used drug classes in our sample were significantly more prevalent in patients with higher overall numbers of drugs (Table 6). This phenomenon, which was also observed in other studies [37], largely reflects the most frequent diagnoses within our cohort. It is, however, noteworthy that of the five most common drug classes (ACE-inhibitors/ARBs, PPIs, statins, platelet-aggregation inhibitors, beta-blockers) only PPIs were directly associated with higher overall numbers of drugs. PPIs are likely to be prescribed in patients with higher drug intake for 'stomach protection' and- as our results confirm- sometimes without clear indication (see above). Thus, PPI use should be questioned and stopped more rigorously.

\section{Strengths and limitations}

A strength of our study is that we assessed not only polypharmacy in combination with a range of demographic and clinical characteristics, but also medication appropriateness and DDIs.

In contrast to other studies [38, 40] we were able to provide information not only about drugs reimbursed by the NHS, but we included all prescribed drugs according to the physicians' EHRs. However, OTC-medications were not assessed because the electronic data extraction was possible only for prescribed drugs which were the only drugs recorded in the EHRs. OTC-drugs could have been additionally collected by questioning the participating patients. However, older-aged patients not always remember all drugs they are taking and brown bag medication reviews with each patient were not feasible within the logistic constraints of the study. Thus, a reliable and complete determination of OTC-drugs was not possible and was therefore a priori excluded.

A German study showed that the prevalence of polypharmacy doubled when OTC-medications were included [53]. Thus, medication use in our study might have been underestimated; however, as in Italy most continuously taken drugs are only available on prescription (except e.g. some low-dose NSAIDs or vitamins), the exclusion of OTC-drugs should not have led to a substantial bias.

Also, new drugs prescribed by specialists and not yet appearing in the GPs' EHRs could have been missed; however, we assume that this does not play a relevant role in our sample as in Italy repeated prescriptions of chronic drug therapies are usually conducted by the GPs.

Our analysis was limited to regularly used drugs and we excluded PRN-medications. Thus, drugs used for acute conditions which might have interacted with other medications and conditions could have been overlooked as well.

We used the Beers criteria instead of European tools which might have been more adapted to the local market [61]; further limitations of the Beers criteria are e.g. missing inclusion of duplicate prescriptions or underuse of indicated drugs [61] as well as not-considering of concomitant conditions and possible drug-disease interactions [62]. Nevertheless, the Beers criteria are widely used which facilitates international comparability; moreover, at the moment of the start of our study, the Beers criteria were more established in Italy compared to other European tools (e.g. STOPP/START criteria) [63, 64] and the last update available for the Italian version of the Beers criteria was published in 2012, recently before the development of the PRIMA study protocol, and was adapted to the Italian market and availabilities of drugs.

In general, information for the GPs regarding patients' real drug consumption needs to be improved especially in case of multiple prescribers and use of OTC-drugs; hereby, electronic medical records merging information about all medical consultations and drug purchases can provide useful support.

We only enrolled patients aged 75 years or more. Thus, the comparability with other studies which often included younger persons is limited. However, this is 
also a strength of our study as the older age groups are less studied up to now although being the most vulnerable cohort of patients [56].

A further strength is the broad inclusion of general practice patients instead of a pre-selected cohort of patients admitted to specific hospital wards. The GP sample as well as the patient sample was consecutively recruited to reduce the risk of selection bias, however, the GP sample was small and can therefore not be considered as representative. The patient sample was somewhat selected as we enrolled only communityliving patients who visited the GP office. More motivated GPs were probably more willing to participate in the study and therefore a selection bias cannot be excluded. Generalisability is further limited by the fact that our results are derived from a northern Italian region and epidemiology of polypharmacy might differ in other populations. However, as stated above, many studies in other European countries have shown similar results which leads to the assumption that our results might be comparable to other national contexts and that the implications of the study can therefore be applicable also to other European countries. As drug prescription patterns vary depending on the respective healthcare system, the results of our study might be more comparable to countries with a similar healthcare system as in Italy (NHS-based).

We a priori only included patients with major polypharmacy and the cut-off applied in our study ( $\geq 8$ drugs) is not very commonly used; thus, comparability with other studies is limited.

We did not assess other covariates with potential independent impact on drug consumption (beyond their possible relationship with patients' age), e.g. frailty or educational level.

Due to the cross-sectional design of this study, no conclusions on causal relationships could be drawn. The publication of the results of the RCT from which this cross-sectional analysis is derived will provide information on causality and outcomes.

\section{Conclusions}

Polypharmacy in our sample was associated with the number of chronic conditions, with a higher number of major DDIs, and with lowered patients' affective status and quality of life. Patients' age, patients' sex as well as GPs' age, GPs' sex and geographical location did not entail a higher risk of polypharmacy. Also, polypharmacy was not related to patients' cognitive status, BMI or to the use of PIMs. Although polypharmacy is not synonymous with inappropriate treatment, it is well-known that it increases the risk of adverse events. Our results indicate that GPs should particularly keep their patients with multiple conditions, reduced health and affective status under surveillance, especially those diagnosed with arthrosis, diabetes, coronary heart disease and COPD, independently from patients' age or sex. Regular medication reviews for these patients by GPs with or without consideration of a multidisciplinary approach (e.g. support of a clinical pharmacist) and/or electronic decision support tool should be implemented as routine measure in general practice care with the prerequisite that the GPs are provided with appropriate time resources and technical/financial support.

We did not identify individual therapeutic subgroups which might be particularly considered as risk drug classes for polypharmacy. However, some drug classes were highly represented among the prescriptions rated as PIMs: especially benzodiazepines should be carefully considered regarding indication, benefit and potential risk. Moreover, patients with antithrombotic/anticoagulant drugs, antidepressants and/or antipsychotics are prone to major DDIs and should be adequately monitored.

In daily practice, the questions supported by our findings should be (a) if the used drugs are necessary and appropriate, (b) if the benefit of a drug outweighs its risk for this specific patient with his/her characteristics, comorbidities and concomitantly used drugs, (c) if the altogether of drugs minimises the risk for undesired harmful consequences, (d) if the applied drug regimen serves to maintain the best possible quality of life, and not least (e) if it is practical and acceptable for the patient.

\section{Abbreviations}

ACE: Angiotensin converting enzyme; ADE: Adverse drug event; ARBs: Angiotensin II receptor antagonists; BMI: Body mass index; CCBs: Calcium channel blockers; 6-CIT: 6-Item Cognitive Impairment Test; COPD: Chronic obstructive pulmonary disease; COX: Cyclooxygenase; DDIs: Drug-drug interactions; EHR(s): Electronic health records; EQ-5D-5L and EQ-VAS: 5-level EQ-5D version (5-Item questionnaire and visual analogue scale measuring health-related quality of life and self-reported health status); 5-GDS: 5-Item Geriatric Depression Scale; GP: General practitioner; ICD9: International Classification of Diseases, Ninth Revision; IQR: Inter-QuartileRange; NHS: National Healthcare System; NSAIDs: Non-steroidal antiinflammatory drugs; OTC-drugs: Over the counter medications (available without prescription); PIM: Potentially inappropriate medication; PPIs: Proton pump inhibitors; PRIMA: Polypharmacy in chronic diseases - Reduction of Inappropriate Medication and Adverse drug events in older populations (study acronym); PRN-drugs: Pro re nata (as needed) medication;

QoL: Quality of life; RCT: Randomized controlled trial; SD: Standard deviation

\section{Acknowledgements}

We thank the participating physicians and patients for their valuable collaboration. We express our acknowledgements to Marialuise Obexer for administrative project management.

\section{Authors' contributions}

Andreas Sönnichsen, Christian Wiedermann, Giuliano Piccoliori and Adolf Engl were responsible for study conceptualisation, design, and scientific supervision. Christian Wiedermann was responsible for funding acquisition and for the project proposal. Giuliano Piccoliori planned the statistical analysis, Marco Sandri analysed and interpreted the collected data. Giuliano Piccoliori, Martina Valentini and Anna Vögele coordinated the project management, data collection and intervention. Sara Schmid and Felix 
Deflorian performed the DDI and PIM analysis of the patients' drug regimens. Angelika Mahlknecht collaborated in the interpretation and analysis of data and wrote the manuscript. All authors commented on previous versions of the manuscript and revised and approved the final manuscript.

\section{Funding}

The study was funded by the Italian Ministry of Health (Office IV of the Head Office for Health Research, Biomedicine and Vigilance of Institutions Office IV; grant number: RF-2010-2319605) and by the Council of Health Service of the Autonomous Province of Bolzano (Office for the Education and Training of Healthcare Professionals). None of the sponsors had access or influence neither on the study conceptualisation, nor on the collection/ analysis/ interpretation of data, nor on writing the manuscript.

\section{Availability of data and materials}

The datasets used during the current study are available from the corresponding author on reasonable request.

\section{Declarations}

\section{Ethics approval and consent to participate}

The study was conducted in compliance with the Declaration of Helsinki and with Italian data protection regulations. Written informed consent was obtained from all participating GPs and patients prior to their inclusion. The cluster-randomised controlled trial from which this cross-sectional analysis is derived was registered with Current Controlled Trials Ltd. (ID ISRC TN: 38449870) on 2013-09-11. Ethical approval was obtained from the responsible Ethics Committee of Bolzano on 2013-05-15, approval number: 42-2013.

\section{Consent for publication}

Not applicable.

\section{Competing interests}

All authors declare that they have no competing interests.

\section{Author details}

'Institute of General Practice and Public Health, College of Health Care Professions, Lorenz Böhler- Straße 13, 39100 Bolzano, Italy. ${ }^{2}$ South Tyrolean Academy of General Practice, Wangergasse 18, 39100 Bolzano, Italy. ${ }^{3}$ Institute of General Practice, Family Medicine and Preventive Medicine, Paracelsus Medical University, Strubergasse 21, 5020 Salzburg, Austria. ${ }^{4}$ Big \& Open Data Innovation Laboratory (BODal-Lab), University of Brescia, Via S. Faustino 74/B, 25122 Brescia, Italy. ${ }^{5}$ Department of General Practice and Family Medicine, Center for Public Health, Medical University of Vienna, Kinderspitalgasse 15/I, 1090 Vienna, Austria. ${ }^{6}$ UMIT - Private University for Health Sciences, Medical Informatics and Technology - Tyrol, Eduard-Wallnöfer-Zentrum 1, 6060 Hall in Tirol, Austria.

\section{Received: 15 January 2021 Accepted: 2 March 2021}

Published online: 20 March 2021

\section{References}

1. Legido-Quigley HPD, Car J, McKee M, Busse R. Clinical guidelines for chronic conditions in the European Union. Geneva: World Health Organization; 2013.

2. Marengoni A, Winblad B, Karp A, Fratiglioni L. Prevalence of chronic diseases and multimorbidity among the elderly population in Sweden. Am J Public Health. 2008;98(7):1198-200.

3. Tragni ESF, Casula M. Elders with multiple chronic conditions: epidemiology and drug use. G Ital Farmacoecon Farmacoutilizzazione. 2014;6(3):12.

4. Maher RL, Hanlon J, Hajjar ER. Clinical consequences of polypharmacy in elderly. Expert Opin Drug Saf. 2014;13(1):57-65.

5. Fialová D, Topinková E, Gambassi G, Finne-Soveri H, Jónsson PV, Carpenter I, et al. Potentially inappropriate medication use among elderly home care patients in Europe. JAMA. 2005;293(11):1348-58.

6. Mahlknecht A, Krisch L, Nestler N, Bauer U, Letz N, Zenz D, et al. Impact of training and structured medication review on medication appropriateness and patient-related outcomes in nursing homes: results from the interventional study InTherAKT. BMC Geriatr. 2019;19(1):257.
7. Midao L, Giardini A, Menditto E, Kardas P, Costa E. Polypharmacy prevalence among older adults based on the survey of health, ageing and retirement in Europe. Arch Gerontol Geriatr. 2018;78:213-20.

8. Holt S, Schmiedl S, Thurmann PA. Potentially inappropriate medications in the elderly: the PRISCUS list. Dtsch Arztebl Int. 2010;107(31-32):543-51.

9. Mannucci PM, Nobili A, Pasina L, Collaborators R. Polypharmacy in older people: lessons from 10 years of experience with the REPOSI register. Intern Emerg Med. 2018;13(8):1191-200.

10. Hanlon JT, Artz MB, Pieper CF, Lindblad Cl, Sloane RJ, Ruby CM, et al. Inappropriate medication use among frail elderly inpatients. Ann Pharmacother. 2004;38(1):9-14.

11. Onder G, Bonassi S, Abbatecola AM, Folino-Gallo P, Lapi F, Marchionni N, et al. High prevalence of poor quality drug prescribing in older individuals: a nationwide report from the Italian Medicines Agency (AIFA). J Gerontol A Biol Sci Med Sci. 2014;69(4):430-7.

12. Fabbietti $P$, Ruggiero C, Sganga F, Fusco S, Mammarella F, Barbini N, et al. Effects of hyperpolypharmacy and potentially inappropriate medications (PIMs) on functional decline in older patients discharged from acute care hospitals. Arch Gerontol Geriatr. 2018;77:158-62.

13. Sganga F, Vetrano DL, Volpato S, Cherubini A, Ruggiero C, Corsonello A, et al. Physical performance measures and polypharmacy among hospitalized older adults: results from the CRIME study. J Nutr Health Aging. 2014;18(6):616-21.

14. Pirmohamed M, James S, Meakin S, Green C, Scott AK, Walley TJ, et al. Adverse drug reactions as cause of admission to hospital: prospective analysis of 18820 patients. BMJ. 2004;329(7456):15-9.

15. Fabbietti P, Di Stefano G, Moresi R, Cassetta L, Di Rosa M, Fimognari F, et al. Impact of potentially inappropriate medications and polypharmacy on 3-month readmission among older patients discharged from acute care hospital: a prospective study. Aging Clin Exp Res. 2018;30(8):977-84.

16. Eurostat. Population structure and ageing. 2016. http://ec.europa.eu/eurosta t/statistics-explained/index.php/Population_structure_and_ageing\#Further_ Eurostat_information. Accessed 5 Feb 2021

17. Office for National Statistics. National Population Projections: 2014-based statistical bulletin. London: Office for National Statistics; 2015.

18. Sonnichsen A, Trampisch US, Rieckert A, Piccoliori G, Vogele A, Flamm M, et al. Polypharmacy in chronic diseases-reduction of inappropriate medication and adverse drug events in older populations by electronic decision support (PRIMA-eDS): study protocol for a randomized controlled trial. Trials. 2016;17:57.

19. Rieckert A, Trampisch US, Klaassen-Mielke R, Drewelow E, Esmail A, Johansson T, et al. Polypharmacy in older patients with chronic diseases: a cross-sectional analysis of factors associated with excessive polypharmacy. BMC Fam Pract. 2018;19(1):113.

20. Garfinkel D, Zur-Gil S, Ben-Israel J. The war against polypharmacy: a new cost-effective geriatric-palliative approach for improving drug therapy in disabled elderly people. Isr Med Assoc J. 2007;9(6):430-4.

21. Herdman M, Gudex C, Lloyd A, Janssen M, Kind P, Parkin D, et al. Development and preliminary testing of the new five-level version of EQ-5D (EQ-5D-5L). Qual Life Res. 2011;20(10):1727-36.

22. Brooke P, Bullock R. Validation of a 6 item cognitive impairment test with a view to primary care usage. Int J Geriatr Psychiatry. 1999;14(11):936-40.

23. Rinaldi P, Mecocci P, Benedetti C, Ercolani S, Bregnocchi M, Menculini G, et al. Validation of the five-item geriatric depression scale in elderly subjects in three different settings. J Am Geriatr Soc. 2003;51(5):694-8.

24. American Geriatrics Society Beers Criteria Update Expert P. American Geriatrics Society updated beers criteria for potentially inappropriate medication use in older adults. J Am Geriatr Soc. 2012;60(4):616-31.

25. Servizio Sanitario Regionale Emilia-Romagna. Appropriatezza prescrittiva nella popolazione anziana - Elenco farmaci potenzialmente inappropriati ed eventuali alternative terapeutiche. Parma: Servizio Sanitario Regionale Emilia-Romagna; 2014.

26. UpToDate ${ }^{\oplus}$ Drugs \& drug interaction database. 2016. https://www.uptodate. com/home/uptodate-drug-interactions-tool. Accessed 6 Mar 2020.

27. EuroQoL. EQ-5D-5L valuation crosswalk index value calculator. 2014. https:// euroqol.org/eq-5d-instruments/eq-5d-5l-about/valuation-standard-valuesets/crosswalk-index-value-calculator/. Accessed 22 Sept 2014.

28. Szende A, Janssen B, Cabases J. Self-reported population health: an international perspective based on EQ-5D. 2014. https://eq-5dpublications. euroqol.org/download?id=0_54006\&fileld=54415. Accessed 2 Apr 2019. 
29. EuroQol Research Foundation. EQ-5D-5L user guide. 2019. https://euroqol. org/publications/user-guides/. Accessed 10 June 2020.

30. Liu Q, Li C, Wanga V, Shepherd BE. Covariate-adjusted Spearman's rank correlation with probability-scale residuals. Biometrics. 2018;74(2):595-605.

31. Koper D, Kamenski G, Flamm M, Bohmdorfer B, Sonnichsen A. Frequency of medication errors in primary care patients with polypharmacy. Fam Pract. 2013;30(3):313-9.

32. Simoes PA, Santiago LM, Mauricio K, Simoes JA. Prevalence of potentially inappropriate medication in the older adult population within primary care in Portugal: a Nationwide cross-sectional study. Patient Prefer Adherence. 2019;13:1569-76.

33. von Buedingen F, Hammer MS, Meid AD, Muller WE, Gerlach FM, Muth C. Changes in prescribed medicines in older patients with multimorbidity and polypharmacy in general practice. BMC Fam Pract. 2018;19(1):131.

34. Arnoldo L, Cattani G, Cojutti P, Pea F, Brusaferro S. Monitoring Polypharmacy in healthcare systems through a multi-setting survey: should we put more attention on long term care facilities? J Public Health Res. 2016;5(3):745.

35. Morin L, Johnell K, Laroche ML, Fastbom J, Wastesson JW. The epidemiology of polypharmacy in older adults: register-based prospective cohort study. Clin Epidemiol. 2018;10:289-98.

36. Arcopinto M, Cataldi M, De Luca V, Orlando V, Simeone G, D'Assante R, et al. Implementing an ICT-based Polypharmacy management program in Italy. Transl Med UniSa. 2017;16:24-9.

37. Schuler J, Duckelmann C, Beindl W, Prinz E, Michalski T, Pichler M. Polypharmacy and inappropriate prescribing in elderly internal-medicine patients in Austria. Wien Klin Wochenschr. 2008:120(23-24):733-41.

38. Raschi E, Piccinni C, Signoretta V, Lionello L, Bonezzi S, Delfino M, et al. Clinically important drug-drug interactions in poly-treated elderly outpatients: a campaign to improve appropriateness in general practice. $\mathrm{Br}$ J Clin Pharmacol. 2015;80(6):1411-20.

39. Bucholc M, O'Kane M, Ashe S, Wong-Lin K. Prescriptive variability of drugs by general practitioners. PLoS One. 2018;13(2):e0189599.

40. Slabaugh SL, Maio V, Templin M, Abouzaid S. Prevalence and risk of polypharmacy among the elderly in an outpatient setting: a retrospective cohort study in the Emilia-Romagna region, Italy. Drugs Aging. 2010;27(12): 1019-28.

41. Mann J. Choice of drug therapy in primary (essential) hypertension. 2020. https://www.uptodate.com/contents/choice-of-drug-therapy-in-primaryessential-hypertension?search=indication\%20ARBs\%20hypertension\&source= search_result\&selectedTitle=1 150\&usage_type=default\&display_rank=1. Accessed 13 Nov 2020.

42. Merel SE, Paauw DS. Common drug side effects and drug-drug interactions in elderly adults in primary care. J Am Geriatr Soc. 2017;65(7):1578-85.

43. Qato DM, Wilder J, Schumm LP, Gillet V, Alexander GC. Changes in prescription and over-the-counter medication and dietary supplement use among older adults in the United States, 2005 vs 2011. JAMA Intern Med. 2016;176(4):473-82.

44. Nobili A, Marengoni A, Tettamanti M, Salerno F, Pasina L, Franchi C, et al. Association between clusters of diseases and polypharmacy in hospitalized elderly patients: results from the REPOSI study. Eur J Intern Med. 2011;22(6): 597-602.

45. Guaraldo L, Cano FG, Damasceno GS, Rozenfeld S. Inappropriate medication use among the elderly: a systematic review of administrative databases. BMC Geriatr. 2011;11:79.

46. By the American Geriatrics Society Beers Criteria Update Expert P. American geriatrics society 2015 updated beers criteria for potentially inappropriate medication use in older adults. J Am Geriatr Soc. 2015;63(11):2227-46.

47. Italian Medicines Agency AIFA (Agenzia Italiana dei Farmaci). 2019. http:// www.agenziafarmaco.gov.it/content/nota-1. Accessed 13 Jan 2021.

48. Schepisi R, Fusco S, Sganga F, Falcone B, Vetrano DL, Abbatecola A, et al. Inappropriate use of proton pump inhibitors in elderly patients discharged from acute care hospitals. J Nutr Health Aging. 2016;20(6):665-70.

49. Weiss V, Nau R, Glaeske G, Hummers E, Himmel W. The interplay of context factors in hypnotic and sedative prescription in primary and secondary carea qualitative study. Eur J Clin Pharmacol. 2019;75(1):87-97.

50. lliffe S, Curran HV, Collins R, Yuen Kee SC, Fletcher S, Woods B. Attitudes to long-term use of benzodiazepine hypnotics by older people in general practice: findings from interviews with service users and providers. Aging Ment Health. 2004;8(3):242-8
51. Muhlack DC, Hoppe LK, Weberpals J, Brenner H, Schöttker B. The Association of potentially inappropriate medication at older age with cardiovascular events and overall mortality: a systematic review and metaanalysis of cohort studies. J Am Med Dir Assoc. 2017;18(3):211-20.

52. Pasina L, Djade CD, Nobili A, Tettamanti M, Franchi C, Salerno F, et al. Drugdrug interactions in a cohort of hospitalized elderly patients. Pharmacoepidemiol Drug Saf. 2013;22(10):1054-60.

53. Junius-Walker $U$, Theile G, Hummers-Pradier E. Prevalence and predictors of polypharmacy among older primary care patients in Germany. Fam Pract. 2007;24(1):14-9.

54. Jyrkka J, Enlund H, Korhonen MJ, Sulkava R, Hartikainen S. Patterns of drug use and factors associated with polypharmacy and excessive polypharmacy in elderly persons: results of the Kuopio 75+ study: a cross-sectional analysis. Drugs Aging. 2009;26(6):493-503.

55. Guthrie B, Makubate B, Hernandez-Santiago V, Dreischulte T. The rising tide of polypharmacy and drug-drug interactions: population database analysis 1995-2010. BMC Med. 2015;13:74.

56. Franchi C, Tettamanti M, Pasina L, Djignefa CD, Fortino I, Bortolotti A, et al. Changes in drug prescribing to Italian community-dwelling elderly people: the EPIFARM-Elderly Project 2000-2010. Eur J Clin Pharmacol. 2014;70(4): $437-43$.

57. Tosato M, Settanni S, Antocicco M, Battaglia M, Corsonello A, Ruggiero C, et al. Pattern of medication use among older inpatients in seven hospitals in Italy: results from the CRiteria to assess appropriate medication use among elderly complex patients (CRIME) project. Curr Drug Saf. 2013;8(2): 98-103.

58. Kim HA, Shin JY, Kim MH, Park BJ. Prevalence and predictors of polypharmacy among Korean elderly. PLoS One. 2014;9(6):e98043.

59. Toepfer S, Bolbrinker J, Konig M, Steinhagen-Thiessen E, Kreutz R, Demuth I. Potentially inappropriate medication in older participants of the Berlin Aging Study II (BASE-II) - sex differences and associations with morbidity and medication use. PLoS One. 2019;14(12):e0226511.

60. Iniesta-Navalon C, Gascon-Canovas JJ, Gama Z, Sanchez-Ruiz JF, GutierrezEstrada EA, De-la-Cruz-Sanchez E, et al. Potential and clinical relevant drugdrug interactions among elderly from nursing homes: a multicentre study in Murcia, Spain. Cien Saude Colet. 2019;24(5):1895-902.

61. Corsonello A, Pranno L, Garasto S, Fabietti P, Bustacchini S, Lattanzio F. Potentially inappropriate medication in elderly hospitalized patients. Drugs Aging. 2009;26(Suppl 1):31-9.

62. Spinewine A, Schmader KE, Barber N, Hughes C, Lapane KL, Swine C, et al. Appropriate prescribing in elderly people: how well can it be measured and optimised? Lancet. 2007;370(9582):173-84.

63. Corsonello A, Onder G, Abbatecola AM, Guffanti EE, Gareri P, Lattanzio F. Explicit criteria for potentially inappropriate medications to reduce the risk of adverse drug reactions in elderly people: from beers to STOPP/START criteria. Drug Saf. 2012;35(Suppl 1):21-8.

64. Ruggiero C, Dell'Aquila G, Gasperini B, Onder G, Lattanzio F, Volpato S, et al. Potentially inappropriate drug prescriptions and risk of hospitalization among older, Italian, nursing home residents: the ULISSE project. Drugs Aging. 2010;27(9):747-58.

\section{Publisher's Note}

Springer Nature remains neutral with regard to jurisdictional claims in published maps and institutional affiliations.

\section{Ready to submit your research? Choose BMC and benefit from:}

- fast, convenient online submission

- thorough peer review by experienced researchers in your field

- rapid publication on acceptance

- support for research data, including large and complex data types

- gold Open Access which fosters wider collaboration and increased citations

- maximum visibility for your research: over $100 \mathrm{M}$ website views per year

At BMC, research is always in progress.

Learn more biomedcentral.com/submissions 